\title{
Microfilament-binding properties of N-terminal extension of the isoform of smooth muscle long myosin light chain kinase
}

\author{
Chun Xiang Yang ${ }^{1,3, *}$, Hua Qun Chen ${ }^{2, *}$, Chen Chen ${ }^{1}$, Wei Ping Yu ${ }^{3}$, Wen Cheng Zhang ${ }^{1}$, Ya Jin Peng ${ }^{1}$, Wei Qi He ${ }^{1}$, \\ Dong Mei Wei ${ }^{1}$, Xiang $\mathrm{Gao}^{1}$, Min Sheng Zhu ${ }^{1,4}$ \\ ${ }^{1}$ Model Animal Research Center and National Key Lab of Medicine, Nanjing University, Nanjing 210061, China ; ${ }^{2}$ College of Life \\ Science, Nanjing Normal University, Nanjing 210097, China; ${ }^{3}$ Medical College, Southeast University, Nanjing 210096, China; \\ ${ }^{4}$ Huadong Research Institute for Medical Biotechnics, Nanjing 210002, China
}

Myosin light chain kinases (MLCK) phosphorylate the regulatory light chain of myosin II in thick filaments and bind to F-actin-containing thin filaments with high affinity. The ability of short myosin light chain kinase (S-MLCK) to bind F-actin is structurally attributed to the DFRXXL regions in its N-terminus. The long myosin light chain kinase (L-MLCK) has two additional DFRXXL motifs and six Ig-like modules in its N-terminal extension. The six Ig-like modules are capable of binding to stress fibers independently. Our results from the imaging analysis demonstrated that the first two intact Ig-like modules (2Ig) in N-terminal extension of L-MLCK is the minimal binding module required for microfilament binding. Binding assay confirmed that F-actin was able to bind 2Ig. Stoichiometries of 2Ig peptide were similar for myofilament or pure F-actin. The binding affinities were slightly lower than 5DFRXXL peptide as reported previously. Similar to DFRXXL peptides, the 2Ig peptide also caused efficient F-actin bundle formation in vitro. In the living cell, over-expression of 2Ig fragment increased "spike"-like protrusion formation with over-bundled F-actin. Our results suggest that L-MLCK may act as a potent F-actin bundling protein via its DFRXXL region and the 2Ig region, implying that L-MLCK plays a role in cytoskeleton organization.

Cell Research (2006) 16:367-376. doi:10.1038/sj.cr.7310047; published online 13 April 2006

Keywords: MLCK, F-actin bundle, Ig-like module

\section{Introduction}

Myosin light chain kinase activates myosin by exclusively phosphorylating Thr-18 and Ser-19 on the N terminus of regulatory light chain (RLC) of myosin II. RLC phosphorylation is sufficient to initiate contraction in smooth muscle, but in striated muscles RLC phosphorylation potentiates the force and speed of contraction [1-3]. Recently, the phosphorylation-dependent myosin II has been increasingly shown to be involved in many processes of non-muscle cells, including cell spreading and migration, extension of neurite growth cone, cytokinesis, cytoskeletal clustering of integrins at focal adhesions, stress

\footnotetext{
*These authors contributed equally to this work. Correspondence: Min Sheng Zhu

Tel: 86-25-58641529; Fax: 86-25-58641500

E-mail: zms@nicemice.cn

Received 26 Sep 2005; revised 10 Jan 2006; accepted 11 Jan 2006, published online 13 Apr 2006
}

fiber formation, platelet shape changes, secretion, exocytosis and transepithelial permeability [4-14]. In vertebrates, there are two MLCK genes at different genomic loci, the skeletal muscle MLCK and smooth muscle MLCK [3]. The smooth muscle MLCK locus expresses three transcripts in a tissue-specific manner due to alternate promoters and produces two MLCK isoforms (short MLCK and long MLCK) and a C-terminal Ig module (telokin) [15-17]. Short MLCK is expressed in smooth muscle, skeletal muscle and non-muscle tissues, whereas long MLCK is expressed in a subset of these tissues (e.g. lung, smooth muscle and embryo tissues). The long MLCK is the predominant isoform expressed in cultured non-muscle cells (e.g. NIH3T3 fibroblast cells, A10 smooth muscle cells and HUVEC endothelial cells) $[18,19]$.

The short MLCK isoform contains a catalytic core, a regulatory segment, three Ig modules, one Fn module as well as a PEVK repeat-rich region and an actin-binding sequence at its $\mathrm{N}$ terminus (3DFRXXL motif) [4]. Long 
MLCK isoform has a molecular mass of $\sim 210 \mathrm{kDa}$, and it is identical to the short MLCK except for a unique N-terminal extension of $\sim 900$ residues. In this extension region, there are six immunoglobin-like modules, a DVRXXL motif and a DFRXXL motif (usually called 2DFRXXL motif). There is evidence that demonstrated the importance of both 5 DFRXXL of long MLCK and 3 DFRXXL of short MLCK in F-actin binding [20, 21]; each DFRXXL may bind to a single actin monomer in an actin filament [22]. Both 5 DFRXXL and 3 DFRXXL are sufficient for MLCK's binding to actin filament with maximal binding stoichiometries [23]. It is hypothesized that binding to F-actin via DFRXXL and to myosin via telokin facilitates RLC phosphorylation by MLCK. When the 5DFRXXL region was deleted, the full length or N-terminus of long MLCK could still bind to stress fiber, implying the existence of a novel binding motif in the extension region [23-25]. Furthermore, Poperechnaya et al. found that, in dividing cells, the extension region was required for MLCK localization to the cleavage furrow. Therefore, they proposed that non-muscle cells utilize different mechanisms for targeting the long MLCK to actomyosin structures during interphase and mitosis [24]. Currently, the exact function of the extension region of long MLCK remains largely unknown. To understand its possible function, it is necessary to elucidate its binding properties. In order to elucidate its possible functions, we performed a detailed characterization of the F-actin binding properties of MLCK's N-terminal extension. We first identified its minimal binding motif, and then determined its binding stoichiometries and ability of F-actin bundle formation. Our results indicate that smMLCK is a potent F-actin bundling protein and may play a role in cytoskeleton organization.

\section{Materials and methods}

\section{Extraction of smooth muscle myofilaments}

The myofilaments were prepared according to the methods described previously [23]. Briefly, chicken gizzards were homogenized in $10 \mathrm{mM}$ MOPS, pH 7.5, $50 \mathrm{mM} \mathrm{NaCl}$, and $1 \mathrm{mM}$ dithiothreitol (DTT) at $\mathrm{pH} 7.5$ with a homogenizer and centrifuged at $17400 \times \mathrm{g}$ for 10 min at $4{ }^{\circ} \mathrm{C}$. To dissociate endogenous MLCK, the resultant pellets were homogenized in $10 \mathrm{ml}$ buffer containing $10 \mathrm{mM}$ Tris-HCl, $\mathrm{pH}$ 7.5, $2 \mathrm{mM}$ EGTA, $2 \mathrm{mM}$ DTT, $50 \mathrm{mM} \mathrm{MgCl}_{2}$ and $3 \%$ Triton $\mathrm{X}-100$. After washing in the same buffer five times, the myofilaments were then washed three times in $10 \mathrm{mM}$ MOPS, $\mathrm{pH} 7.5,2$ mM EGTA, 2 mM DTT and stored in the same buffer.

\section{F-actin cross-linking}

G-actin from rabbit skeletal muscle was prepared according to the methods described previously [26]. Purified G-actin was determined by non-reduced SDS-PAGE and the purity was higher than $98 \%$. $\mathrm{F}$-actin was polymerized by dialysis with polymerizing buffer ( 50 $\mathrm{mM} \mathrm{KCl}, 1 \mathrm{mM} \mathrm{MgCl}$, $1 \mathrm{mM}$ EGTA, $15 \mathrm{mM}$ imidazole, $0.2 \mathrm{mM}$
$\mathrm{Na}_{2}$ ATP, $1 \mathrm{mM}$ DTT, $1 \mathrm{mM}$ Tris-HCl, $\mathrm{pH}$ 7.5) for $30 \mathrm{~min}$ at room temperature and centrifuged at a low speed $(12000 \times \mathrm{g})$ for $10 \mathrm{~min}$ to eliminate potential pellets formed by dialysis. Under our condition, there was no obvious F-actin detected in the pellet fraction. Increasing concentrations of 1 Ig or 2Ig peptide (1-4 $\mu \mathrm{M})$, which was dialyzed against MOPS binding buffer (10 mM MOPS, 100 $\mathrm{mM} \mathrm{NaCl}, 1 \mathrm{mM}$ EGTA and $1 \mathrm{mM}$ dithiothreitol), were incubated with $6 \mu \mathrm{M}$ F-actin at $25^{\circ} \mathrm{C}$ for $1 \mathrm{~h}$ in the MOPS binding buffer. The reaction mixtures were centrifuged at $12000 \times \mathrm{g}$ for $30 \mathrm{~min}$. The proteins in pellet and supernatant fractions were separated by SDS-PAGE and stained with Coomassie brilliant blue R250. The relative actin contents in the pellet and supernatant fractions were quantified by densitometry.

\section{Construction of $M L C K-G F P$ variants}

As described in our previous report [23], pEGFP-MLCK210 plasmid contains the full length of L-MLCK gene driven by CMV promoter, and pEGFP(N-term)MLCK plasmid contains the $\mathrm{N}$ terminal six Ig-like motifs of long MLCK. In order to construct pEGFP(2DFRXXL)MLCK plasmid, a fragment of long MLCK (from 2352 to $2802 \mathrm{nt}$ ) was amplified by PCR reaction with primer pairs: 5' GCA AAA TGA AGA TAT CTT CAC ACT G $3^{\prime}$ and 5' GGA TCC CCT TGC TCG GCT GGG ATT TC 3', and subcloned into TOPO vector and then digested by EcoR V/BamH I. The digested fragment was ligated to pEGFP(N-term)MLCK plasmid digested with the same enzymes; pEGFP-427 MLCK was made by removing the region from EcoR I to BamH I site (1 277-2 639nt) of pEGFP(N-term)MLCK, and then self-ligated with the vector after blunting the ends. For pEGFP-4Ig construction, pEGFP(N-term)MLCK was digested with EcoR I and then ligated with an adaptor made by annealing two oligos, 5' AAT TGT TAT GGC 3' and 5' AAT TGC CAT AAC 3'. In order to make a mutant vector containing the first two Ig-like modules (1-251nt) of long MLCK, pEGFP-427MLCK was digested with EcoR I/Sph I and the released fragment was subcloned into $\mathrm{pGEM} 3 \mathrm{z}$ and then released by EcoR I/Hind III, in which the Hind III end was blunted by a klenow fragment. The fragment was ligated with pEGFP (N-term) MLCK vector, in which pre-existing MLCK fragment was deleted by digestion with $E c o \mathrm{R} \mathrm{I/BamH} \mathrm{I} \mathrm{(BamH} \mathrm{I} \mathrm{end} \mathrm{was} \mathrm{blunted).} \mathrm{The}$ resultant plasmid was called pEGFP-251MLCK. To make pEGFP125 MLCK plasmid, an MLCK fragment (from 362 to $1281 \mathrm{nt}$ ) was obtained by PCR with primer pairs 5' GAA TTC TTG AAG AAA TAC AG 3' and 5' GGA TCC ATT CCC AAA GGC TC 3', and subcloned into TOPO vector and then the $E c o R \mathrm{I} / B a m H$ I fragment was inserted into pEGFP(N-term)MLCK vector digested with $E c o$ R I/BamH I. Both pEGFP-200MLCK containing the intact first Iglike module and half of the second Ig-like module (1-200 aa) and pEGFP108MLCK containing the intact first Ig-like module only (1-108 aa) within the N-terminus were constructed by subcloning MLCK fragment amplified from MLCK cDNA.

All the constructs above were sequenced and confirmed to contain correct sequences of reading frames. The expressed products were verified by Western blotting with anti-GFP monoclonal antibody, as shown in Figure 1.

\section{Construction of expression plasmids of MLCK fragments \\ To express HA-tagged MLCK peptide, the oligos of 5' GAT CCA TGT ACC CAT ACG ATG TTC CAG ATT ACG CTC TTT G 3' and 5' AGC TCAAAG AGC GTAATC TGG AAC ATC GTA TGG GTA}


CAT G $3^{\prime}$ were annealed and inserted into pET28a vector digested with Hind III and BamH I. The resultant plasmid pET-HA was used as an expression vector in the following expression experiments. 1Ig (1-128aa) or 2Ig (1-208aa) of MLCK was obtained by polymerase chain reaction with primers pairs of $1 \mathrm{Ig}, 5^{\prime}$ CAT ATG GGG GAT GTT AAA CTG 3' and 5' GGA TCC TAG GCT GTA TTT CTT C 3'; 2Ig, 5' CAT ATG GGG GAT GTT AAA CTG 3' and 5' GGA TCC CTT ATC TGG ACC TTG AAC 3'. The PCR products were harbored in pM18-T vector (Takara Inc.) and the fragments were released by Nde I and Hind III restriction enzymes, and then inserted into pETHA plasmid digested with Nde I and Hind III. The final expression plasmids were named $\mathrm{pET}-1 \mathrm{Ig}$ and $\mathrm{pET}-2 \mathrm{Ig}$.

\section{Protein expression and purification}

Recombinant plasmids harboring $1 \mathrm{Ig}$ or $2 \mathrm{Ig}$ were transformed into BL21(DE3) cells and induced by $0.5 \mathrm{mM}$ isopropyl-1-thioD-galactopyranoside (IPTG) as described [27]. The bacteria were harvested by centrifugation at $8000 \times \mathrm{g}$ for $10 \mathrm{~min}$, resuspended in lysis buffer (20 mM TrisHCl, pH 8.0, 0.1\% lysozyme) and lysed by ultrasonication at $4^{\circ} \mathrm{C}$. The suspension was then centrifuged at $12000 \times g$ for $10 \mathrm{~min}$ at $4{ }^{\circ} \mathrm{C}$. The proteins were purified by using histidine affinity column (His $\times$ Tag) chromatography (Novagen) and dialyzed against MOPS buffer (10 mM MOPS, pH 7.5, $50 \mathrm{mM} \mathrm{NaCl}$,
$1 \mathrm{mM} \mathrm{MgCl} 2,1 \mathrm{mM}$ DTT, $0.1 \mathrm{mM}$ EGTA) and stored at $-80^{\circ} \mathrm{C}$. The protein concentrations were quantified with BCA kit (Pierce Inc.), the protein purities were determined by SDS-PAGE and the purity was always higher than $95 \%$.

\section{Co-sedimentation assay [23, 28]}

Various amounts of recombinant peptides were mixed with 0.7 $\mathrm{mg} / \mathrm{ml}$ myofilament containing $6 \mu \mathrm{M}$ of $\mathrm{F}$-actin in a final volume of $100 \mu \mathrm{l}$ binding buffer ( $10 \mathrm{mM}$ MOPS, $\mathrm{pH} 7.0,50 \mathrm{mM} \mathrm{NaCl}, 1 \mathrm{mM}$ $\mathrm{MgCl}_{2}, 1 \mathrm{mM}$ DTT, $0.1 \mathrm{mM}$ EGTA, $10 \%$ glycerol and $0.5 \mathrm{mg} / \mathrm{ml}$ bovine serum albumin). The mixture was incubated for $30 \mathrm{~min}$ at 4 ${ }^{\circ} \mathrm{C}$. The reaction mixtures were then centrifuged at $17400 \times g$ for 5 $\min$ at $4^{\circ} \mathrm{C}$. The pellets were washed briefly and resuspended in 100 $\mu \mathrm{l}$ binding buffer. Equal amounts of samples $(30 \mu \mathrm{l})$ from supernatant fraction and pellet suspension were subjected to SDS-PAGE and Western blot assay. The primary antibody for detecting the peptides was anti-HA monoclonal antibody (Chemicon Inc.) and the secondary antibody we used was HRP-labeled goat anti mouse antibody (Sigma Inc.). The peptides in both supernatant and pellet fractions were detected by Western blotting assay. The positive bands were visualized by ECL at different exposure times and quantified by densitometry. To prevent the effect of overexposure on quantification accuracy, standard linear regression curves against density values

A

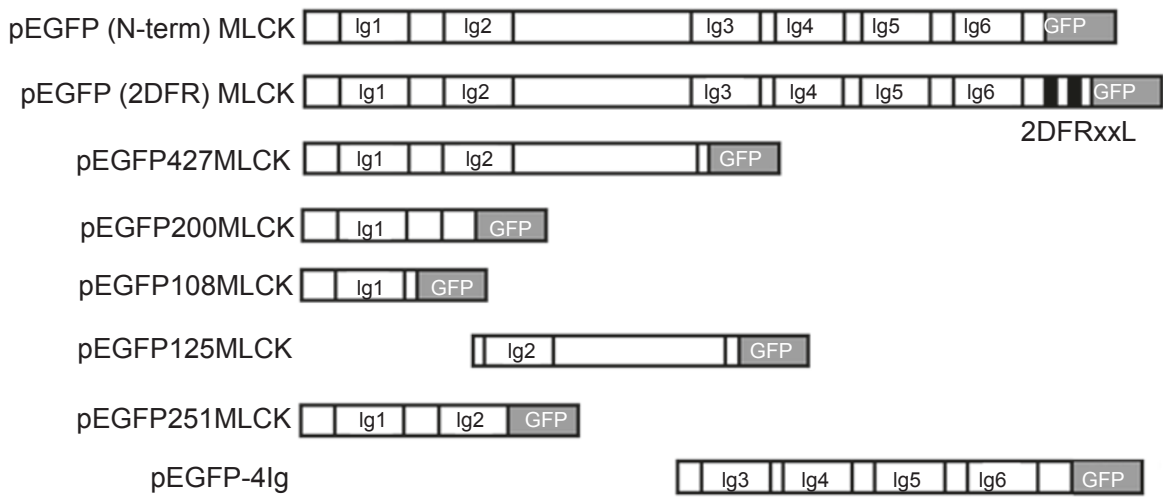

B

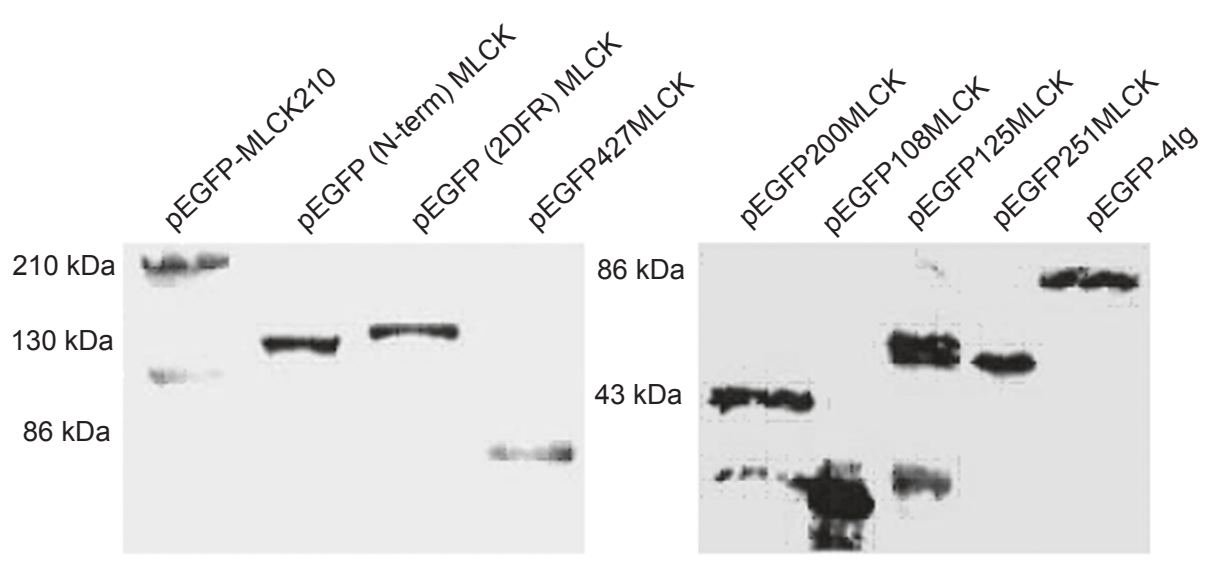

Figure 1 Schematic representation of long MLCK truncated mutants and fusion proteins. (A) N-terminal extension of long MLCK contains two DFRXXL motifs and six Ig-like modules, which are numbered in frames. GFP fusion proteins are shown in gray solid frames. All GFP fused MLCK mutants are driven by CMV promoter. (B) Mutant constructs were transfected into COS-7 cells and expressed recombinant proteins were visualized by Western blot assay with anti-GFP monoclonal antibody. 
and amounts of peptides were plotted, and optimized with different exposure times. The optimized curves were used as standard quantification curves. Values of KD and Bmax were estimated for individual experiments subjected to Scatchard analysis. To determine stoichiometry of binding to F-actin, similar co-sedimentation assay was performed.

\section{Cell culture and transfection}

Twenty-four hours before transfection, NIH3T3 cells were seeded into $60 \mathrm{~mm}$ tissue culture dishes at a density of $3 \times 10^{5}$ in $5 \mathrm{ml}$ DMEM medium containing $5 \%$ fetal bovine serum and $100 \mathrm{U} / \mathrm{ml}$ penicillin-streptomycin. LipofectAmine Plus (Invitrogen Co.) was used for transfection according to the manufacturer's protocol. COS-7 cells were transfected with $3 \mu \mathrm{g}$ plasmid and $4 \mu$ l Fugene- 6 (Roche Biochemicals) according to the manufacturer's manual.

\section{Cell imaging}

Fluorescence imaging of transfected NIH3T3 cells with different MLCK-GFP variant constructs was performed using a fluorescence microscope as described previously [21, 23]. Twelve-bit images were obtained with a CCD camera (Quantix Photometrics; Tucson, AZ) and Oncorimage software (Oncor; Gaithersburg, MD). Narrow bandpass interference filters (Omega; Brattleboro, VT) were used to detect GFP (490 nm excitation and $520 \mathrm{~nm}$ emission) and rhodamine (550 $\mathrm{nm}$ excitation and $575 \mathrm{~nm}$ emission). During image collection, cells were kept at $37^{\circ} \mathrm{C}$ in an open thermal-controlled chamber (Custom Scientific; Dallas, TX). Cells were subsequently treated with a buffer containing $0.02 \%$ saponin in $20 \mathrm{mM}$ PIPES, pH $6.8,4 \mathrm{mM}$ EGTA, $90 \mathrm{mM} \mathrm{K}^{+}$gluconate, $5.3 \mathrm{mM} \mathrm{Na}_{2} \mathrm{ATP}, 5 \mathrm{mM} \mathrm{MgSO}_{4}, 0.1 \%$ bovine serum albumin, $0.1 \mathrm{mM}$ phenylmethylsulfonyl fluoride, 10 $\mu \mathrm{g} / \mathrm{ml}$ leupeptin and $10 \mu \mathrm{g} / \mathrm{ml}$ of aprotinin for $4 \mathrm{~min}$ at $37^{\circ} \mathrm{C}$. The cells were then washed three times with the buffer in the absence of saponin, and then images were obtained.

\section{Localization of microfilament binding in cells}

Culture medium was removed from dishes and replaced with serum-free medium containing $1 \mu \mathrm{M}$ latrunculin $\mathrm{B}$. The medium was removed again and washed once with pre-warmed PBS. The cells were made permeable with PBS containing $0.1 \%$ Triton X100 for $1 \mathrm{~min}$ and fixed with $1 \%$ paraformaldehyde for $30 \mathrm{~min}$. After blocking with $1 \%$ glycine and $1 \%$ BSA solution sequentially, MLCK peptides were incubated with the cells and the bound peptides were determined with anti-HA monoclonal antibody and visualized by FITC-labeled secondary antibody. For $1 \mathrm{Ig}$ binding experiment used as negative control, the same methods were carried out. To determine F-actin fibers, rhodamine-labeled phalloidin was stained for 10 min and washed twice with PBS before confocal microscopy (Leica TCS-SL).

\section{Morphological observation of F-actin bundle formation}

To determine bundle formation morphologically under a confocal microscope, a modified method was used [29]. Briefly, $4 \mu 1$ of $5 \mu \mathrm{M}$ rhodamine-labeled phalloidin in methanol was dropped on a coverslip and allowed to dry for $1 \mathrm{~h}$. A thick layer of vaseline was applied around the phalloidin. F-actin was polymerized in polymerizing buffer for $1 \mathrm{~h}$ at room temperature. Various amounts of recombinant peptides were mixed with $6 \mu \mathrm{M}$ of F-actin. After reaction for $1 \mathrm{~h}$ at room temperature, the mixture was loaded on top of dried rhodaminephalloidin. The mixture was polymerized for $1 \mathrm{~h}$ at room temperature and examined with a confocal microscope (Leica TCS-SL). For 3D imaging, a stack of 20-100 frames was collected with an interval of $100 \mathrm{~nm}$ between adjacent slices. For determining the F-actin bundles under an electron microscope, the previously reported method [29, 30] was used. Briefly, various amounts of peptide were incubated with $6 \mu \mathrm{mol} / \mathrm{L}$ of polymerized $\mathrm{F}$-actin for $1 \mathrm{~h}$ at room temperature. Mixtures were pelleted down at $14000 \times \mathrm{g}$ for $20 \mathrm{~min}$. The supernatant was discarded, and the pellet was resuspended in the original volume of polymerization buffer. A $10 \mu \mathrm{l}$ of liquid specimen was placed onto a sheet of parafilm and undisturbed for $30 \mathrm{~s}$. After removing excess fluid with filter paper and washing the grid in water, the specimen was negatively stained with $2 \%$ uranyl acetate for $1 \mathrm{~min}$ and allowed to dry. Specimens were observed in a transmission electron microscope (JECL JEM-1200EX).

\section{Results}

\section{Intact $2 \mathrm{Ig}$ fragment of MLCK N-terminus is sufficient for microfilament binding}

Using imaging analysis, previous data showed that L-MLCK's N-terminus that lacked DFRXXL motifs also bound microfilament in cells, suggesting the existence of a novel-binding motif [23-25]. To identify the minimum microfilament-binding region, we made a set of expression vectors that encode truncated $\mathrm{N}$-terminal extension fragments (Figure 1A). COS-7 cells were used for the transfection experiments, and the recombinant proteins in cell lysate were confirmed by Western blot assay with anti-GFP monoclonal antibody (Figure 1B).

To determine the binding ability of each truncated peptide to stress fibers, plasmid DNA constructs were transfected into NIH3T3 cells and results showed that pEGFP251MLCK transfected cells exhibited obvious fluorescent stress fibers, whereas pEGFP108MLCK and

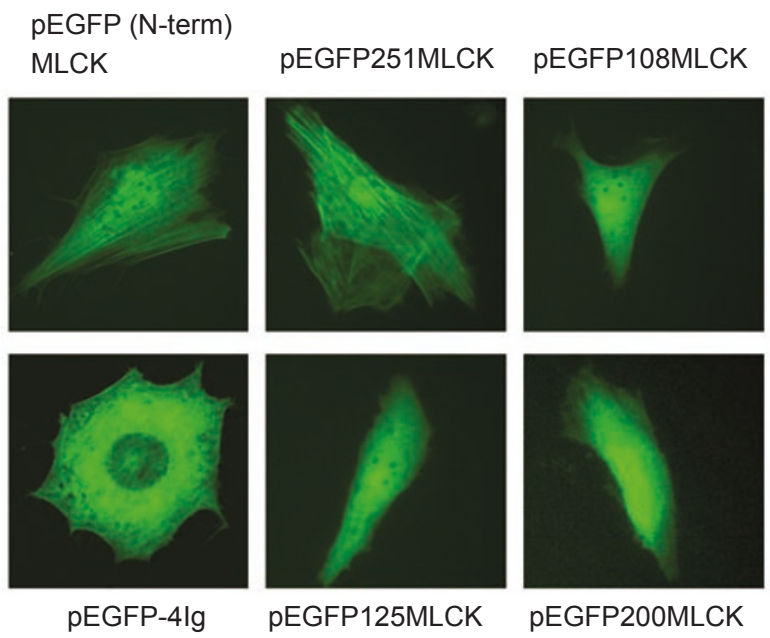

Figure 2 Expression of long MLCK mutants in NIH3T3 cells. Each plasmid DNA was transfected into NIH3T3 cells with lipofectAmine plus reagent and the green fibers were determined under a fluorescent microscope $48 \mathrm{~h}$ after transfection. 
pEGFP200MLCK did not (Figure 2). The fluorescence pattern in the stress fiber was indistinguishable to that induced by pEGFP(N-term)MLCK (Figure 2) as well as pEGFP(2DFR)MLCK containing six Ig-like motifs plus the 2DFRXXL region of long smMLCK (data not shown). Similar results were obtained in transfected $\mathrm{C} 2 \mathrm{C} 12$ myoblast cells and A10 smooth muscle cells (data not shown). This showed that the two intact Ig domains in the N-terminus were sufficient for binding to stress fiber.

To determine the specificity of 2Ig binding ability, the cells were made permeable by saponin treatment to release the unbound fluorescent protein from cytoplasm. The fluorescence in the stress fiber of the pEGFP251MLCKtransfected cells remained unchanged when the cells were made permeable, and the GFP signal was co-localized with the cellular microfilament visualized by rhodaminelabeled phalloidin. In contrast, the cells transfected with pEGFP108MLCK or pEGFP4Ig plasmid showed no GFP fluorescence in the stress fiber in both intact and permeable conditions (Figure 3).

\section{Disassembling of microfilament blocks $2 \mathrm{Ig}$ binding to filament}

The co-localization of the first two Ig fragment (pEGFP251MLCK) and six Ig fragment (pEGFP(N-term)MLCK)

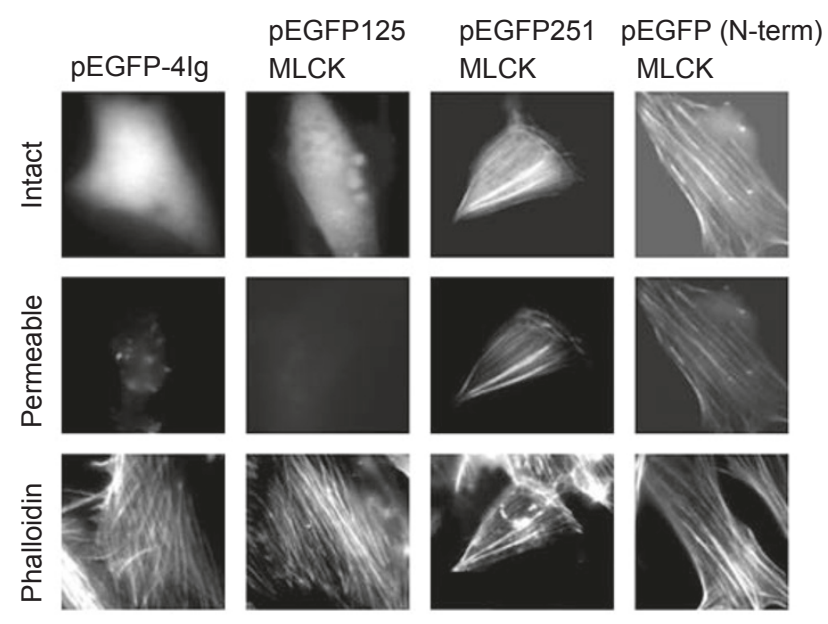

Figure 3 Binding of truncated long MLCK and its mutants to microfilaments. NIH3T3 fibroblasts were transfected with L-MLCK-GFP fusion constructs or with various mutant constructs that include different Ig-like modules. pEGFP-4Ig contains the third, fourth, fifth and sixth Ig-like module in N-terminal extension; pEGFP125MLCK contains the second Ig-like module and pEGFP251MLCK contains the first two Ig-like modules; pEGFP(N-term)MLCK has six Ig-like modules. The cells in the middle and bottom rows were made permeable with saponin treatment, whereas the middle row shows GFP fluorescence and the bottom row shows rhodamine-labeled phalloidin to reveal actin-containing stress fibers. Representative images for at least four experiments are shown.

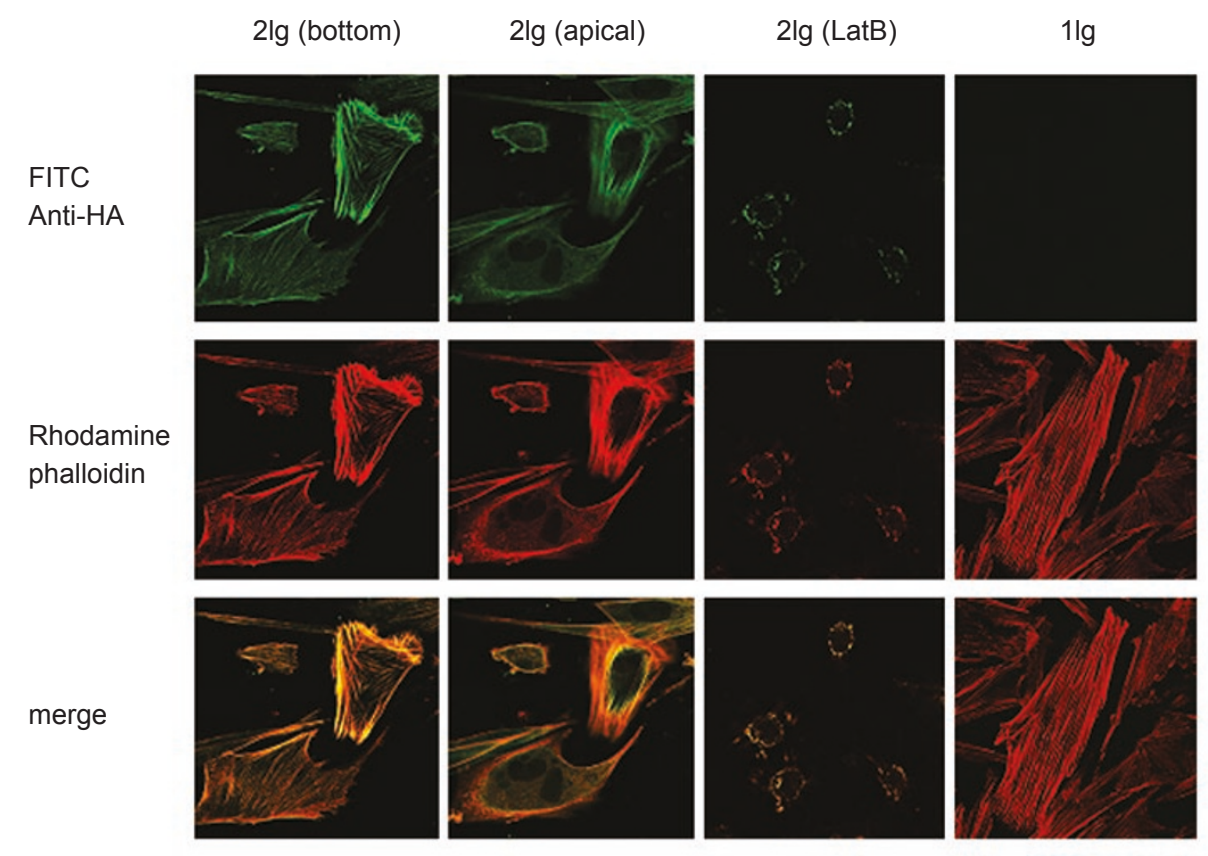

Figure 4 Intracellular localization of recombinant MLCK-HA peptide bound in NIH3T3 fibroblast cells. NIH 3T3 cells were treated with complete medium (as a control) and $1 \mu \mathrm{M}$ latrunculin-B (Lat B) for $1 \mathrm{~h}$. Then, the cells were fixed with paraformaldehyde and blocked with $1 \mathrm{M}$ glycine and $0.1 \%$ BSA sequentially, and incubated with HA-tagged MLCK peptides. The bound peptides were visualized with anti-HA monoclonal antibody and FITC-labeled antibody. The top row shows peptide-bound filaments, the middle row shows actin-containing microfilament and the bottom row displays merge layers of the green and red filaments. 
with microfilaments indicated that F-actin is the binding target of the 2Ig fragment of L-MLCK. To confirm this, F-actin in the microfilament of NIH3T3 cell was disassembled by latrunculin B treatment and we found that the binding of 2Ig peptide to the fibers was disrupted. No phalloidin-stained stress fibers was found in latrunculin B-treated cells, although amorphous F-actin aggregates could be observed (Figure 4).

Because microfilament is mainly distributed in the stress fiber, cell cortex and cell membrane edge, which could be observed in different cellular layers under a confocal microscope, we wanted to determine whether the 2Ig-bound microfilament also showed a region-specific pattern. At the bottom of cells, GFP signal (2Ig binding) were perfectly co-localized with red stress fibers (Figure 4). At the middle layer, green fluorescence was distributed at the membrane edge and cortex region of cells and also perfectly co-localized with F-actin fibers. These results indicated that 2Ig binding is not stress fiber specific, but is general to all F-actin containing microfilament in different regions of the cell. Recombinant 1Ig peptide of MLCK did not bind F-actin microfilament of the cell (Figure 4).

2 Ig peptides bind to F-actin filaments with high affinity

To quantify the myofilament-binding and F-actin-binding ability of $2 \mathrm{Ig}$ protein in vitro, recombinant $2 \mathrm{Ig}$ and

A

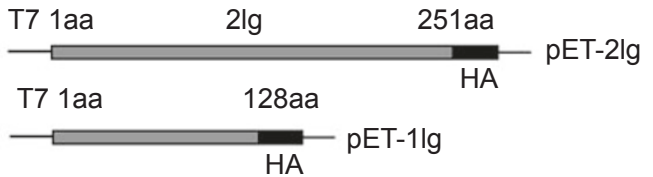

B

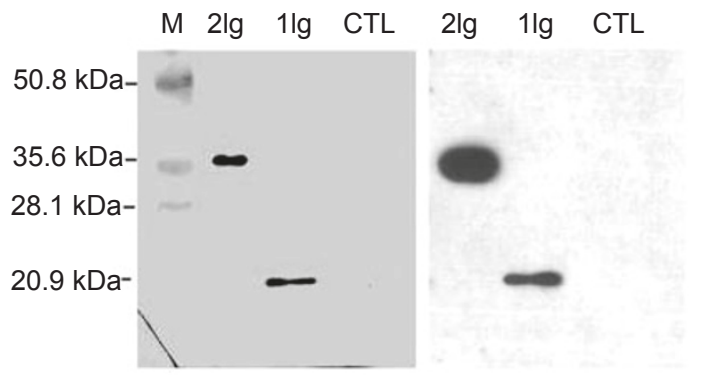

Figure 5 Recombinant expression of 2Ig fragment of MLCK in E.coli. (A) Long MLCK fragments $1 \operatorname{Ig}(1-128 \mathrm{aa})$ and $2 \operatorname{Ig}(1-251 \mathrm{aa})$ were fused with HA-Tag and subcloned into pET28a. The resultant expressive plasmids pET-2Ig and pET-1Ig were transformed to BL21 (DE3) cells and induced by IPTG. (B) Purified proteins were resolved in SDS-PAGE gels and transferred to PVDF membrane. Western blot assay and Coomassie brilliant blue R250 staining were performed sequentially in the same membrane. Lane $\mathrm{M}$ indicates standard molecular weight of proteins; lanes $2 \mathrm{Ig}$ and $1 \mathrm{Ig}$ represent $2 \mathrm{Ig}$ and $1 \mathrm{Ig}$ peptides, respectively; lane CTL indicates control protein purified from BL21 cell lysate with His $\times$ Tag column.
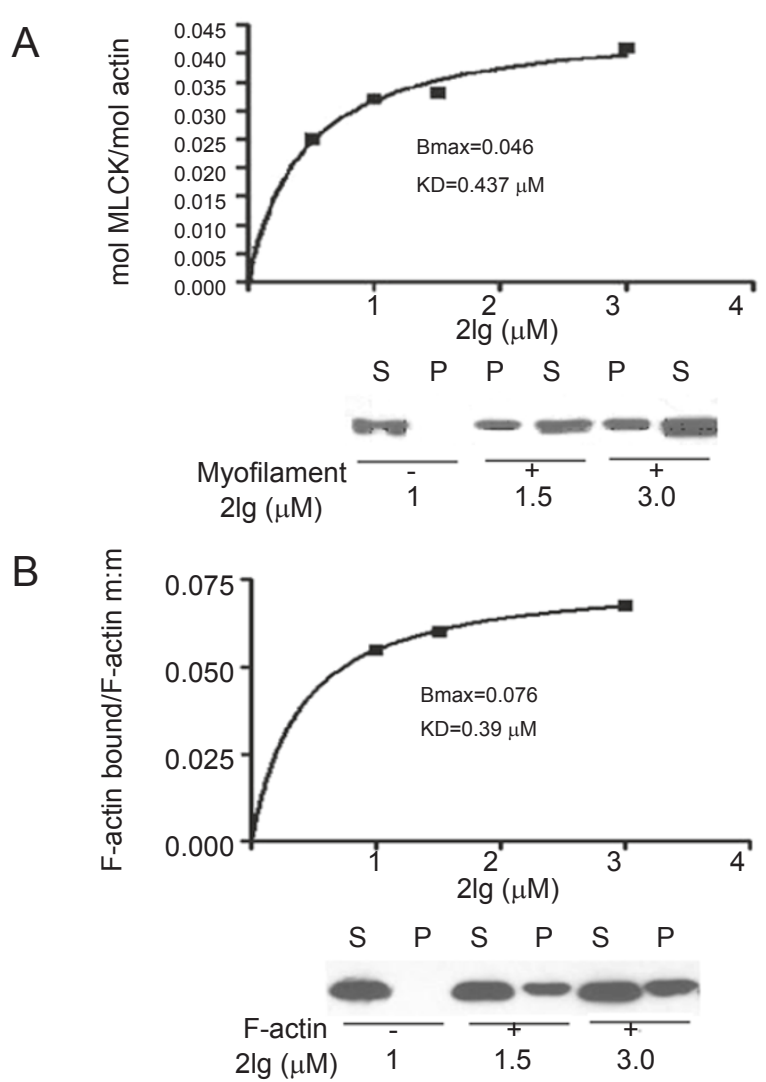

Figure 6 Binding ability of 2Ig peptide to myofilament. Increasing concentrations of $2 \mathrm{Ig}$ peptide were mixed with $6 \mu \mathrm{M}$ actin in myofilament (A) or F-actin (B) for co-sedimentation assay as described in the Materials and methods section. The amount of bound 2Ig peptide (mol MLCK $/ \mathrm{mol}$ actin) is plotted against the 2Ig peptide concentration. Data representative of at least four independent experiments are shown. S: peptide in supernatant fraction; P: peptide in pellet fraction.

1 Ig peptides tagged by HA were expressed in E. coli. BL21(DE3) and the recombinant peptides were confirmed by SDS-PAGE and Western blotting with anti-HA monoclonal antibody, as shown in Figure 5 (A and B). These peptides were subject to sedimentation assay. 2 Ig peptide co-sedimented with myofilament, and the maximal amount of $2 \mathrm{Ig}$ binding was about $0.06 \mathrm{~mol}$ of peptide $/ \mathrm{mol}$ of actin lower than that of 5DFRXXL peptide $(0.21 \mathrm{~mol}$ of peptide/mol of actin, [23]). As shown in Figure 6A and Table 1, the results also showed that $2 \operatorname{Ig}$ has a higher KD value $(0.45 \mu \mathrm{M})$ than 5 DFRXXL peptide $(0.25 \mu \mathrm{M}) .1 \mathrm{Ig}$ peptide did not co-sediment with myofilament. When concentrations of 1 Ig peptide were increased to $3 \mu \mathrm{M}$ in the assay mixture, weak binding could be detected and it was consistent with typical non-specific binding curve in terms of linear regression curve without saturation binding (data not shown). 
Table 1 Binding properties of 2Ig MLCK peptides

\begin{tabular}{lll}
\hline Filaments & KD & Bmax \\
\hline F-actin & $0.40 \pm 0.10$ & $0.08 \pm 0.02$ \\
Myofilament & $0.45 \pm 0.05$ & $0.06 \pm 0.02$ \\
\hline
\end{tabular}

KD: mM; Bmax: mol peptide/mol actin

Binding properties of $2 \mathrm{Ig}$ MLCK peptide. The $2 \mathrm{Ig}$ peptide was incubated with detergent-washed smooth muscle myofilaments or F-actin as described in Materials and Methods section. After sedimentation the amount of bound peptide was measured for KD and

Bmax calculation. The values represent the mean \pm S.E. for at least four individual experiments.

In addition to actin, myofilaments contain a set of associated proteins, such as myosin, actinin and fascin. To rule out the possibility that $2 \mathrm{Ig}$ peptide binds to myofilament indirectly by interacting with other filament-associated protein, pure polymerized F-actin was used for sedimentation assay. Similar results of stoichiometry of binding were obtained. The Bmax and KD are $0.08 \mathrm{~mol}$ of peptide/mol of actin and $0.4 \mu \mathrm{M}$, respectively (Figure $6 \mathrm{~B}$ and Table 1 ). The similarity in the KD and maximal amount of peptide binding suggested that the myofilament-binding capability of $2 \operatorname{Ig}$ peptide was due to its ability to bind F-actin and that F-actin was 2Ig's binding target.

\section{Actin cross-linking}

To assess the ability of $2 \mathrm{Ig}$ peptide to cross-link actin filaments, low-speed centrifugation assay was conducted as described previously [23]. Increasing concentrations of peptides were incubated with F-actin at a fixed concentration $(6 \mu \mathrm{M})$. Both F-actin and peptides could only be detected in the sediments when the actin filaments were cross-linked with peptides and formed F-actin bundles. As shown in Figure 7 (A and B), $3 \mu \mathrm{M}$ 2Ig peptide crosslinked about $60 \%$ of $\mathrm{F}$-actin. In contrast, when adding $1 \mathrm{Ig}$ peptide in the incubation buffer, F-actin in pellet fraction was not detectable (data not shown). Myofilament solution contained pre-existing filament bundles that pelleted down easily at low-speed centrifugation as mentioned above. Under our experimental condition, when myofilament was added to the binding buffer for $1 \mathrm{~h}$, about $40 \%$ $\mathrm{F}$-actin was disassociated from the myofilament bundles and remained in supernatant fraction during centrifugation. To determine the ability of 2 Ig peptide to cross-link the disassociated F-actin in myofilament, we conducted a similar cross-linking experiment by adding peptides to a fixed concentration of myofilament (containing $6 \mu \mathrm{M}$ of actin). As shown in Figure 7A and 7B, after adding 2Ig peptide to myofilament reaction mixture, the amount of F-actin was increased in the pellet and decreased in the supernatant as the peptide concentrations were increased.
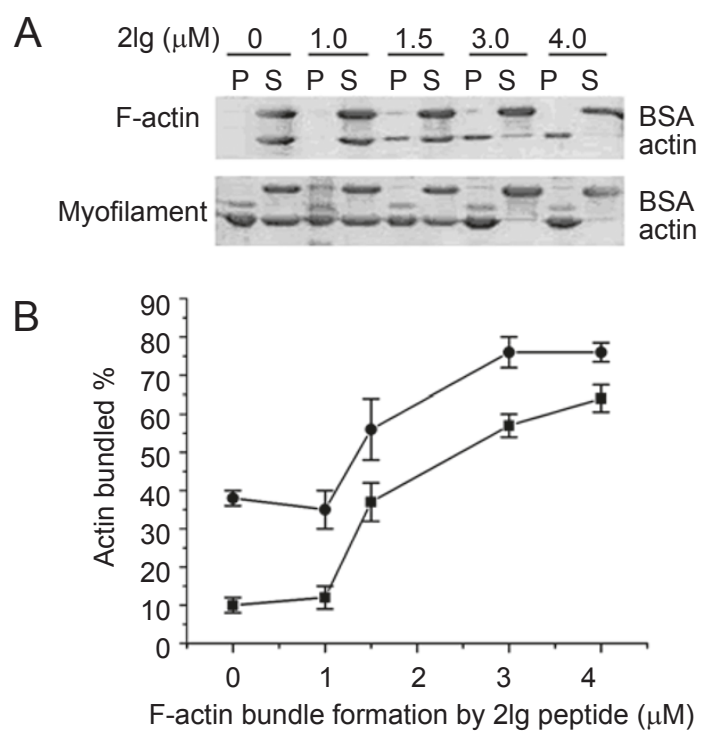

Figure 7 Bundle formation of F-actin induced by 2Ig peptide. (A) $6 \mu \mathrm{M}$ F-actin or myofilaments were incubated with increasing concentrations of 2 Ig peptide and cross-linking bundles were determined by low-speed centrifugation. F-actin content in the pellet and supernatant fractions was analyzed by Coomassie blue staining and densitometry quantification. Each point corresponds to the mean $\pm \mathrm{SE}$ ( $n=3)$. $\mathbf{m}$ : F-actin; •: myofilament. (B) A typical Coomassie staining for cross-linking assay. P: pellet fraction; S: supernatant fraction.
A

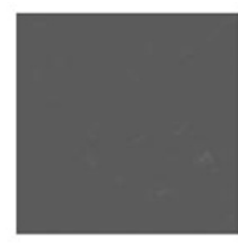

$\mathrm{R}=0$

B

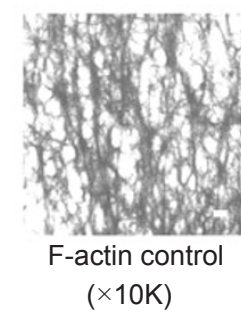

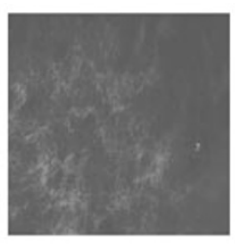

$\mathrm{R}=0.5$

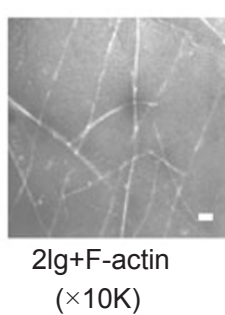

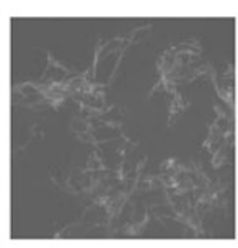

$\mathrm{R}=1$

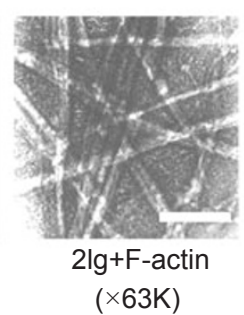

Figure 8 Morphology of F-actin bundles under microscopes. (A) 6 $\mu \mathrm{M}$ F-actin was mixed with $3 \mu \mathrm{M}$ or $6 \mu \mathrm{M}$ of $2 \mathrm{Ig}$ peptide and then loaded on a rhodamine-labeled phalloidin pre-treated slide. The Factin bundles formed were examined under a confocal microscope. $R=0, R=0.5$ and $R=1$ indicates that 0,3 and $6 \mu \mathrm{M} 2 \operatorname{Ig}$ peptide were added to $6 \mu \mathrm{M}$ F-actin, respectively. (B) $6 \mu \mathrm{M}$ F-actin was mixed with $6 \mu \mathrm{M} 2 \mathrm{Ig}$ peptide and the bundles were examined under an electron microscope as described in the Materials and methods section. These experiments were repeated three times and similar results were obtained. Scale bar=100 nm. 


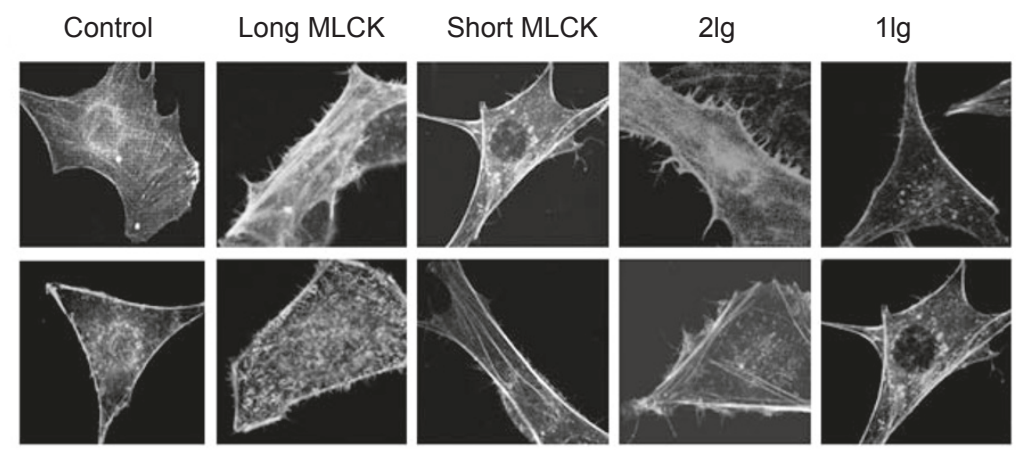

Figure 9 Increased spike formation elicited by 2 Ig. Colon 26 cells were transfected with $4 \mu$ g plasmid DNA by lipofectamine2000. The transfected cells were fixed with paraformaldehyde and stained with rhodamine-phalloidin and examined under a confocal microscope. Control: Colon 26 cells transfected with pEGFPC3; 1Ig: Colon 26 cells transfected with pEGFP108MLCK; 2Ig: Colon 26 cells transfected with pEGFP251MLCK; long MLCK: Colon 26 cells transfected with pEGFP-MLCK210; short MLCK: Colon 26 cells transfected with short MLCK-EGFP [23].

Similarly, there was no increase in the amount of F-actin in pellet when 1Ig peptides were added (data not shown). These results indicated that $2 \mathrm{Ig}$ peptide might contain at least two F-actin binding regions and they were sufficient for cross-linking actin filaments.

To determine the morphology of F-actin bundle formation, polymerized F-actin was mixed with different molar ratios of 2 Ig peptide and the F-actin bundles could be visualized with rhodamine-labeled phalloidin and observed under a confocal microscope. The bundle formation could be observed clearly, and the amount and thickness of the bundles were increased as $\mathrm{R}(\mathrm{R}$, molar ratio of $2 \mathrm{Ig}$ peptide against F-action) increased (Figure 8A). Similar results were obtained by an electron microscope (TEM) (Figure $8 \mathrm{~B}$ ). When $R=1$, the diameter of F-actin bundle was estimated to be about $20 \mathrm{~nm}$.

Taken together, the above results demonstrated that $2 \mathrm{Ig}$ peptide contained at least two actin-binding motifs, was sufficient for cross-linking actin filaments, and could act as an F-actin bundling protein.

Over-expression of $2 \mathrm{Ig}$ increases spike-like filopodia formation

Filopodium is a spike-like protrusion that contains compact F-actin bundle structure; it functions as a sensor of the local environment and has a mechanical role in cell protrusion [31]. For example, the F-actin bundle protein Fascin could elicit spike-like filopodia formation, which is important for cell migration [32]. In order to determine if 2Ig was able to induce spike formation, we transfected MLCK plasmids into Colon 26 cells and examined the cell morphology. $24 \mathrm{~h}$ after transfection, the cells were fixed with paraformaldehyde and stained with rhodamine-phalloidin. pEGFP251MLCK (2Ig) transfected cells showed clear spikes while pEGFP108MLCK (1Ig) transfected cells did not (Figure 9). Both short MLCK and long MLCK transfected cells showed spike formation, although long MLCK could induce spike formation to a greater extent (Figure 9). In fact, figures from our previous report also showed such spikes upon closer examination[23]. Under our experimental condition, 2Ig expressing cells exhibited more numerous and longer spikes in comparison to those transfected with short and long MLCKs. The reason may be that the expression level of 2Ig is higher than that of short or long MLCK. In contrast, the Colon 26 control cells showed smooth edges (Figure 9).

\section{Discussion}

It is proposed that MLCK binds to microfilament by targeting F-actin via the DFRXXL region, and binds to myosin filament by targeting light chain via Ig module at the very C-terminus of MLCK, which results in anchoring of MLCK for efficient phosphorylation of the regulatory myosin light chain [4]. The six Ig-like modules in the Nterminal extension of long MLCK are also sufficient for binding to microfilament. We systematically truncated the six Ig-like regions, and found that the first two Ig motifs were the minimal binding module. This conclusion was also strengthened by the other binding experiments described in this report. Further separation of these two motifs would lead to loss of microfilament-binding activity. As there is a host of F-actin binding proteins associated with microfilament, whether the 2Ig module binds to the fiber via F-actin directly or via F-actin-associated protein indirectly is not clear. Our results demonstrated that the $2 \operatorname{Ig}$ peptide not only bound to microfilament in cells, but also bound to purified F-actin and elicited F-actin bundle formation in vitro. When the cells in culture were treated with latrunculin B that disassembles actin filaments by sequestering 
monomeric actin, no binding was observed between $2 \operatorname{Ig}$ peptide and the microfilament. Thus, these results suggested that $2 \operatorname{Ig}$ was associated with microfilament by binding to F-actin directly.

The organization and behavior of actin filaments inside cells are determined by a remarkable array of actin-binding proteins (ABP). As a group of ABP, cross-linking (or bundling) proteins including fimbrin, villin, fascin and actinin play important roles in rearranging the three-dimensional organization of actin filaments. Short MLCK is capable of cross-linking F-actin fibers and forming bundles via its Factin binding site(s). It could be characterized as a member of actin-bundling proteins [23, 30, 33-35]. Interestingly, our report that N-terminal extension of long MLCK was also capable of cross-linking F-actin and had an ability to elicit $\mathrm{F}$-actin bundle formation in vitro further strengthens this categorization. In the in vivo experiment, over-expression of 2Ig protein led to increased "spike" formation around the cell membrane. Such F-actin-rich spikes were very similar to filopodia that also contain dense F-actin bundles. Therefore, we propose that MLCK may function as an Factin bundling protein, because of its bundling formation ability from both $2 \mathrm{Ig}$ modules in $\mathrm{N}$-terminal extension. As filopodia are important for cell migration and cytoskeleton re-organization, our result suggests a possible structural (cytoskeletal) role for MLCK in cell migration besides its well-known catalytic functions.

Both 3DFRXXL of short MLCK and 5DFRXXL of long MLCK are sufficient for F-actin binding. 3DFRXXL peptide showed a similar binding affinity to 5DFRXXL peptide. The similar KD values were determined to be about $0.16-0.22 \mu \mathrm{M}$ in our previous experiments [23]. In this report, the $\mathrm{KD}$ of $2 \mathrm{Ig}$ was estimated as $0.40 \mu \mathrm{M}$. This result indicates an apparent binding affinity for $2 \mathrm{Ig}$, but still lower than the 5DFRXXL region. As there is 5DFRXXLlike structure in the $2 \mathrm{Ig}$ region by sequence comparison, we speculate that the interaction of the $\mathrm{N}$-terminus of long MLCK with $\mathrm{F}$-actin has a binding mechanism different from that of DFRXXL. This is consistent with the fact that the full length of long MLCK has a greater affinity for microfilaments than short MLCK [24, 25].

While we were completing the binding assay for this study, Kudryashov et al. reported that the N-terminus of long MLCK was able to bind to both F-actin and microtubules, and they proposed that long MLCK might be a potential cytoskeleton integrator through its unique $\mathrm{N}$-terminal domain [36]. Surprisingly, the KD of N-terminal peptide for F-actin binding in their report was estimated to be 7.2 $\mu \mathrm{M}$ and Bmax was over $1.5 \mathrm{~mol} / \mathrm{mol}$ of actin. Similarly, the $\mathrm{KD}$ value for microtubule binding was also up to $7 \mu \mathrm{M}$ and Bmax was over $1.5 \mathrm{~mol} / \mathrm{mol}$ of tubulin. Such binding parameters do not provide a convincing explanation for $2 \mathrm{Ig}$ binding properties because such weak binding affinity is unlikely to contribute to efficient binding to stress fiber and bundle formation. We suspect that the discrepancy between their report and our data is caused by different methods of synthesizing peptide as well as of different binding buffers. As there are several basic amino acids within the first Ig-like module of the N-terminus of long MLCK, the recombinant peptide can be easily precipitated. In our experiments, the recombinant peptides were purified from a soluble extract of E.coli induced at low temperature and the purified proteins were equilibrated thoroughly in MOPS binding buffer. This treatment may prevent autonomous pellet formation in our binding assay, as the pellet formation could result in artificial high $\mathrm{KD}$ and high Bmax. It is conceivable that the same could also take place for the microtubules binding assay. In fact, the pellets were formed even when no F-actin was added to the reaction as shown in Figure $6 \mathrm{~A}$ of their paper [36]. In addition, we failed to detect bound $2 \mathrm{Ig}$ peptide in tubulin pellets under our experimental condition (data not shown).

In summary, we characterized the binding properties of $\mathrm{N}$-terminal extension of L-MLCK and found that the $2 \mathrm{Ig}$ module is the minimal binding region. L-MLCK could be a potent $\mathrm{F}$-actin bundle protein through its function in the 2Ig module. These results may advance our understanding of the cellular functions of L-MLCK.

\section{Acknowledgements}

We thank Dr James Stull and Dr Kristine Kamm for their generous help and instructive suggestions, Dr Qing Shun Zhao for valuable discussions on the manuscripts, Dr Jiong Chen for editing the manuscript, Min Yan Yu for cell culture help and Peng Yu Gu for technical assistance on confocal microscopy. This work was supported by National Natural Science Foundation of China (No. 30470852) and the National Gongguan Project of China (21001BA710B).

\section{References}

1 Kamm KE, Stull JT. The function of myosin and myosin light chain kinase phosphorylation in smooth muscle. Annu Rev Pharmacol Toxicol 1985; 25:593-620.

2 Hartshorne DJ. In: Johnson LR, eds. Physiology of the Gastrointestinal Tract. Raven Press: New York 1987:423-482.

3 Stull JT, Lin PJ, Krueger JK, Trewhella J, Zhi G. Myosin light chain kinase: functional domains and structural motifs. Acta Physiol Scand 1998; 164:471-482.

4 Kamm KE, and Stull JT. Dedicated myosin light chain kinases with diverse cellular functions. J Biol Chem 2001; 276:45274530 .

5 Schoenwaelder SM, Burridge K. Bidirectional signaling between the cytoskeleton and integrins. Curr Opin Cell Biol 1999; 11:274286. 
6 Bresnick AR. Molecular mechanisms of nonmuscle myosin-II regulation. Curr Opin Cell Biol 1999; 11:26-33.

7 Sato M, Tani E, Fujikawa H, Kaibuchi K. Involvement of Rhokinase-mediated phosphorylation of myosin light chain in enhancement of cerebral vasospasm. Circ Res 2000; 87:195-200.

8 van Nieuw Amerongen GP, van Delft S, Vermeer MA, Collard JG, van Hinsbergh VW. Activation of RhoA by thrombin in endothelial hyperpermeability: role of Rho kinase and protein tyrosine kinases. Circ Res 2000; 87:335-340.

9 Jung C, Chylinski TM, Pimenta A, Ortiz D, Shea TB. Neurofilament transport is dependent on actin and myosin. J Neurosci 2004; 24:9486-9496.

10 Clayburgh DR, Rosen S, Witkowski ED, et al. A differentiationdependent splice variant of myosin light chain kinase, MLCK1, regulates epithelial tight junction permeability. J Biol Chem 2004; 279:55506-55513.

11 Tran QK, Watanabe H, Zhang XX, Takahashi T, Ohno R. Involvement of myosin light-chain kinase in chloride-sensitive Ca2+ influx in porcine aortic endothelial cells. Cardiovasc Res 1999; 44:623-631.

12 Szaszi K, Kurashima K, Kapus A, et al. RhoA and rho kinase regulate the epithelial $\mathrm{Na}+/ \mathrm{H}+$ exchanger $\mathrm{NHE} 3$. Role of myosin light chain phosphorylation. J Biol Chem 2000; 275:2859928606.

13 Aromolaran AS, Albert AP, and Large WA. Evidence for myosin light chain kinase mediating noradrenaline-evoked cation current in rabbit portal vein myocytes. J Physiol 2000; 524:853-863.

14 Ammit AJ, Armour CL, Black JL. Smooth-Muscle Myosin Light-Chain Kinase Content Is Increased in Human Sensitized Airways. Am J Respir Crit Care Med 2000; 161:257-263.

15 Birukov KG, Schavocky JP, Shirinsky VP, et al. Organization of the genetic locus for chicken myosin light chain kinase is complex: multiple proteins are encoded and exhibit differential expression and localization. J Cell Biochem 1998; 70:402-413.

16 Watterson DM, Schavocky JP, Guo L, et al. Analysis of the kinase-related protein gene found at human chromosome $3 \mathrm{q} 21$ in a multi-gene cluster: organization, expression, alternative splicing, and polymorphic marker. J Cell Biochem 1999; 75:481-491.

17 Smith AF, Bigsby RM, Word RA, Herring NT. A 310-bp minimal promoter mediates smooth muscle cell-specific expression of telokin. Am J Physiol 1998; 274:C1188-1195.

18 Blue EK, Goeckeler ZM, Jin Y, et al. 220- and 130-kDa MLCKs have distinct tissue distributions and intracellular localization patterns. Am J Physiol 2002; 282:C451-460.

19 Herring BP, Dixon S, Gallagher PJ. Smooth muscle myosin light chain kinase expression in cardiac and skeletal muscle. Am J Physiol 2000; 279:C1656-1664.

20 Smith L, Su X, Lin P, Zhi G, Stull JT. Identification of a novel actin binding motif in smooth muscle myosin light chain kinase. J Biol Chem 1999; 274:29433-29438.

21 Lin P, Luby-Phelps K, Stull JT. Properties of filament-bound myosin light chain kinase. J Biol Chem 1999; 274:5987-5994.

22 Smith L, Stull JT. Myosin light chain kinase binding to actin filaments. FEBS Lett 2000; 480:298-300.

23 Smith L, Parizi-Robinson M, Zhu MS, et al. Properties of long myosin light chain kinase binding to $\mathrm{F}$-actin in vitro and in vivo. J Biol Chem 2002; 277:35597-35604.

24 Poperechnaya A, Varlamova O, Lin PJ, Stull JT, Bresnick AR. Localization and activity of myosin light chain kinase isoforms during the cell. J Cell Biol 2000; 151:697-708.

25 Kudryashov DS, Chibalina MV, Birukov KG, et al. Unique sequence of a high molecular weight myosin light chain kinase is involved in interaction with actin cytoskeleton. FEBS Lett 1999; 463:67-71.

26 Pollard TD, Goldberg I, and Schwarz WH. Nucleotide exchange, structure, and mechanical properties of filaments assembled from ATP-actin and ADP-actin. J Biol Chem 1992; 267:2033920345.

27 Zhu MS, Pan Y, Chen HQ, et al. Induction of SARS-nucleoprotein-speicfic immune response by use of DNA Vaccine. Immunol Lett 2004; 92:237-243.

28 Shirinsky VP, Vorotnikov AV, Birukov KG, et al. A kinase-related protein stabilizes unphosphorylated smooth muscle myosin minifilaments in the presence of ATP. J Biol Chem 1993; 268:1657816583.

29 Shin JH, Gardel ML, Mahadevan L, Matsudaira P, Weitz DA. Relating microstructure to rheology of a bundled and cross-linked F-actin network in vitro. PNAS 2004; 101:9636-9641.

30 Yang CX, Wei DM, Chen C, Yu WP, Zhu MS. 5DFRXXL region of long myosin light chain kinase causes F-actin bundle formation. Chin Sci Bull 2005; 50: 2044-2050.

31 Mogilner A, Rubinstein B. The physics of filopodial protrusion. Biophysics Journal 2005; 89:782-795.

32 Yamashiro S, Yamakita Y, Ono S, Matsumura F. Fascin, an actinbundling protein, induces membrane protrusions and increases cell motility of epithelial cells. Mol Biol Chem 1998; 9:9931006.

33 Kudryashov DS, Chibalina MV, Birukov KG, et al. Unique sequence of a high molecular weight myosin light chain kinase is involved in interaction with actin cytoskeleton. FEBS Lett 1999; 463:67-71.

34 Hayakawa K, Okagaki T, Higashi-Fujime S, Kohama K. Bundling of actin filaments by myosin light chain kinase from smooth muscle. Biochem Biophys Res Commun 1994; 199:786-791.

35 Hayakawa K, Okagaki T, Ye LH, et al. Characterization of the myosin light chain kinase from smooth muscle as an actin-binding protein that assembles actin filaments in vitro. Biochim. Biophys Acta 1999; 1450:12-14.

36 Kudryashov DS, Stepanova OV, Vilitkevich EL, et al. Myosin light chain kinase $(210 \mathrm{kDa})$ is a potential cytoskeleton integrator through its unique N-terminal domain. Exp Cell Res 2004; 298:407-417.

Edited by Zhong Cheng Zheng 